\title{
Effect of metoclopramide on transport in the small intestine of the dog
}

\author{
JOHN TINKER AND ALAN G. COX \\ From the Department of Surgery, Royal Postgraduate Medical School and Hammersmith Hospital, \\ Ducane Road, London
}

SUMMARY A method is described for the simultaneous measurement of flow rate, mean transit time, and volume of the intestine during the transport of fluid through a segment of the small intestine of the dog.

Using this method, the effect of metoclopramide on motility of the small bowel was assessed. The drug resulted in a decrease in transit time and volume in the test segment of bowel while the flow rate remained constant. This finding suggests that metoclopramide-stimulated contraction of intestinal smooth muscle.

The pharmacological actions of metoclopramide (N-(diethylaminoethyl)-2-methoxy-4-amino-5-chlorobenzamide) were described in 1964 by JustinBesançon and Laville, 1964a and b; Justin-Besançon, Laville, and Thominet, 1964). They found that this compound stimulated gastrointestinal smooth muscle and was a potent anti-emetic. Further animal studies by Jacoby and Brodie (1967) did not reveal any of the general parasympathomimetic activity usually associated with agents which increase intestinal motility, suggesting that metoclopramide acted by direct stimulation of intramural cholinergic neurones.

Earlier studies on the effect of metoclopramide on small intestinal motility have been confined to analysis of intraluminal pressure changes or to estimates of transit time by radiological techniques (James and Hume, 1968). Neither method provides a direct measurement of movement of intestinal contents. The present study had two purposes. The first was to evaluate a dye dilution method described by Dillard, Eastman, and Fordtran (1965) for the simultaneous measurement of flow rate, transit time, and intestinal volume in the jejunum. The second was to investigate the effect of metoclopramide on small bowel motility measured by the dye dilution method.

\section{METHOD}

The following method of perfusing a segment of jejunum was employed in three conscious dogs.

OPERATION TO ESTABLISH PERfuSION CIRCUIT A doublelumen tube (Fig. 1) was inserted through a metal gas- trostomy cannula so that the distal opening of one lumen (for perfusion) lay at the duodeno-jejunal flexure and the opening of the other lumen (for injection of dye) lay $15 \mathrm{~cm}$ distal to this.

Then $60 \mathrm{~cm}$ of small intestine was measured distal to the tip of the double-lumen tube. The jejunum was transected at this point and re-anastomosed end to side, with the distal open end of bowel fixed to a second metal cannula brought out through the abdominal wall. In this way, a small Roux loop was constructed. Then, bypassing a small Foley catheter through the jejunal cannula, inflation of the bulb occluded the lumen of the distal

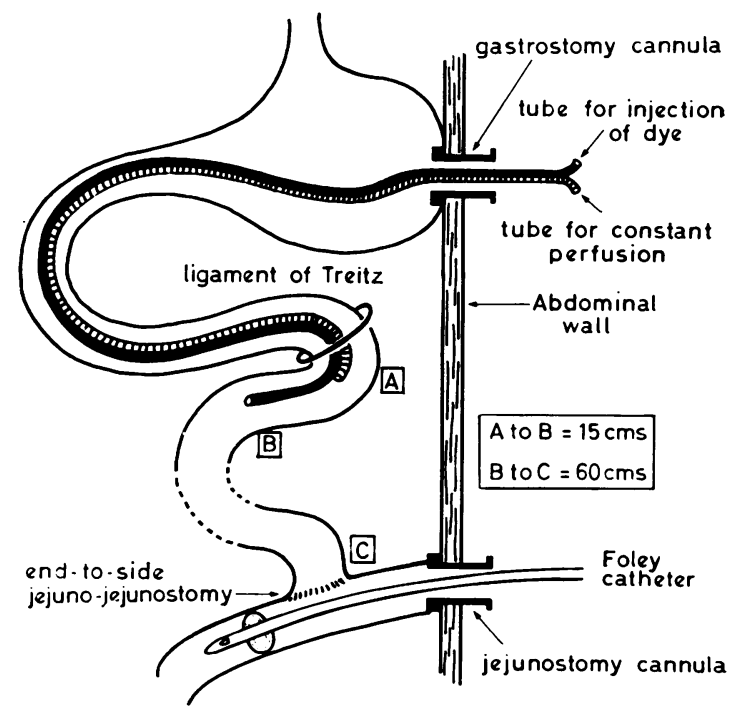

FIG. 1. Diagram of the perfusion circuit. 
jejunum and allowed collection of virtually all fluid passing along the proximal jejunum.

PERFUSING SOLUTION This consisted of an isotonic electrolyte solution containing $1.25 \%$ mannitol (to inhibit net water absorption), $1 \%$ polyethylene glycol (a non-absorbable marker), and sodium chloride to bring the osmolarity to 290 milliosmols per kilogram of solution.

Polyethylene glycol was included in the solution so that the degree of concentration or dilution of the perfusing solution in the intestinal segment could be measured and an average flow rate at the distal collecting site determined by calculations independent of those made from the dye dilution curves (see below).

PERFUSION EXPERIMENT Starting three weeks after operation the following perfusion experiments were carried out with each dog in a Pavlov stand. All experiments were done after an overnight fast.

Perfusion of the isotonic solution at a rate of $14 \mathrm{ml} / \mathrm{min}$ was commenced through the perfusion lumen of the double-lumen tube until a steady collection from the jejunal cannula was obtained, usually within 30 to 45 minutes. After reaching this 'steady state', $1 \mathrm{ml}$ containing $50 \mathrm{mg}$ of a non-absorbable dye, bromsulphthalein (BSP), was injected instantaneously through the injection lumen of the double-lumen tube. Collections of effluent from the jejunal cannula were then made at one-minute intervals, by simple gravity drainage into a container, for a total of 30 minutes. Metoclopramide or saline in equal volumes was injected intravenously during the first few seconds of the perfusion period.

CHEMICAL ANALYSIS (1) The concentration of BSP in each one-minute sample was measured spectrophotometrically after alkalinization of appropriately diluted samples of intestinal fluid. (2) The polyethylene glycol content of the pooled samples was measured by the method of Hyden (1955).

Calculation Dye dilution curves (Fig. 2) were constructed from the results of the BSP estimations using the method described and interpreted in a publication by Zierler (1958). The area under the curve, expressed in units of concentration (mg per $\mathrm{ml}$ ) multiplied by time, was calculated.

The flow rate (the volume of fluid passing a point in the intestine per unit time) at the collection site is equal to the amount of BSP $(\mathrm{mg})$ injected divided by the area under the curve. The mean transit time (the average time required for fluid to travel from a proximal to a distal part of the small intestine) was calculated from the following equation:-

Mean transit time

$\bumpeq$ sum of (each concentration of BSP $\times$ its transit time). sum of each concentration of BSP

Then, flow rate multiplied by mean transit time gives the volume of fluid contained in the intestinal segment between the points where dye was injected and the samples were collected.

Calculations of flow rates independent of the dye dilution method were made by calculating the ratio of the concentration of polyethylene glycol (PEG) in the infused solution to that in the pooled samples obtained from the collection cannula using the following formula :Mean flow rate $=\frac{(\text { PEG }) \text { infused } \times \text { infusion rate }}{(\text { PEG }) \text { collected }}$

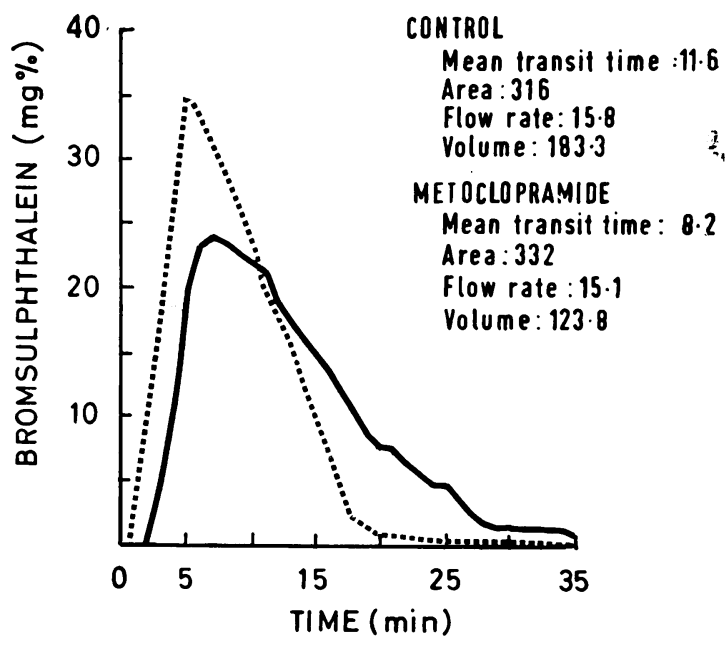

FIG. 2. Examples of dye dilution curves showing the effect of saline and metoclopramide. The continuous line represents the result with saline and the interrupted line that of metoclopramide. The key shows the values which can be derived from these curves.

APPLICATION OF METHOD Using the method described, the effect of metoclopramide on the flow rate, transit time, and intestinal volume was measured.

Beecham Research Laboratories supplied two sets of identical ampoules marked ' $A$ ' and ' $B$ ', each labelled as containing metoclopramide $10 \mathrm{mg} / 2 \mathrm{ml}$. Only one set, unknown to the observer, contained metoclopramide and the other contained normal saline. When the project had been finished the code was broken: ampoules ' $A$ ' contained metoclopramide and ampoules ' $B$ ' saline.

In each dog the effects of an intravenous injection of drug $\mathbf{A}$, drug $\mathbf{B}$, and an equal volume of saline on small bowel motility, were measured. In a previous study by Hakuhara, Nakayama, Fakuda, Siina, and Neya (1966), using a Thisry-Vella fistula as the experimental model, the optimal intravenous dose of metoclopramide appeared to be of the order of 1 to $5 \mathrm{mg} / \mathrm{kg}$ body weight. In the present study $1.5 \mathrm{mg} / \mathrm{kg}$ body weight was selected. Five separate studies with each of the three agents were carried out on two dogs. In the third dog, four studies with saline and three each with drug A and drug B were carried out making a total of 40 perfusion experiments.

Student's $t$ test was used to calculate significances of differences between the effects of the three agents injected. $P$ values less than 0.05 were regarded as representing a statistically significant difference. 
TABLE I

\begin{tabular}{|c|c|c|c|c|c|}
\hline \multirow[b]{2}{*}{ Dog No. } & \multicolumn{5}{|c|}{ MEASUREMENTS OF INTESTINAL MOTILITY ${ }^{1}$} \\
\hline & Infusion & No. of Studies & $\begin{array}{l}\text { Mean Transit Time } \\
\text { (minutes) }\end{array}$ & $\begin{array}{l}\text { Mean Flow Rate } \\
(\mathrm{ml} / \mathrm{min})\end{array}$ & $\begin{array}{l}\text { Mean Volume of } \\
\text { Perfused Segment }\end{array}$ \\
\hline 1 & $\left\{\begin{array}{l}\text { Saline } \\
\text { A (metoclopramide) } \\
\text { B (saline) }\end{array}\right.$ & $\begin{array}{l}5 \\
5 \\
5\end{array}$ & $\begin{array}{l}6 \cdot 乞 4 \pm 0 \cdot 2 \\
3 \cdot 9^{2} \pm 0 \cdot 2 \\
7 \cdot 2 \pm 0 \cdot 5\end{array}$ & $\begin{array}{l}19 \cdot 4 \pm 0 \cdot 7 \\
17 \cdot 3 \pm 0 \cdot 8 \\
17 \cdot 6 \pm 1 \cdot 4\end{array}$ & $\begin{array}{r}117 \cdot 6 \pm 5 \cdot 7 \\
67 \cdot 3^{2} \pm 3 \cdot 5 \\
126 \cdot 3 \pm 7 \cdot 1\end{array}$ \\
\hline 2 & $\left\{\begin{array}{l}\text { Saline } \\
\text { A (metoclopramide) } \\
\text { B (saline) }\end{array}\right.$ & $\begin{array}{l}4 \\
3 \\
3\end{array}$ & $\begin{array}{l}6 \cdot 3 \pm 2 \cdot 0 \\
3 \cdot 4^{2} \pm 0 \cdot 3 \\
8 \cdot 5 \pm 0 \cdot 2\end{array}$ & $\begin{array}{l}17 \cdot 2 \pm 1 \cdot 7 \\
18 \cdot 3 \pm 2 \cdot 5 \\
19 \cdot 4 \pm 2 \cdot 3\end{array}$ & $\begin{aligned} 161 \cdot 8 & \pm 16 \cdot 03 \\
82 \cdot 3^{2} & \pm 8 \cdot 6 \\
165 \cdot 0 & \pm 21 \cdot 3\end{aligned}$ \\
\hline 3 & $\left\{\begin{array}{l}\text { Saline } \\
\text { A (metoclopramide) } \\
\text { B (saline) }\end{array}\right.$ & $\begin{array}{l}5 \\
5 \\
5\end{array}$ & $\begin{array}{cc}12 \cdot 3 & \pm 2 \cdot 2 \\
7 \cdot 8^{2} & \pm 0 \cdot 5 \\
12 \cdot 3 & \pm 1 \cdot 2\end{array}$ & $\begin{array}{l}15 \cdot 6 \pm 2 \cdot 8 \\
14 \cdot 4 \pm 1 \cdot 3 \\
16 \cdot 9 \pm 2 \cdot 4\end{array}$ & $\begin{array}{l}193 \cdot 6 \pm 60 \cdot 7 \\
112 \cdot 1^{2} \pm 9 \cdot 4 \\
198 \cdot 8 \pm 45 \cdot 2\end{array}$ \\
\hline
\end{tabular}

${ }^{1}$ Means \pm SE.

${ }^{2}$ These values are significantly lower $(\mathrm{P}<0.05)$ than those with saline and $\mathrm{B}$ None of the other mean results in each column is significantly different for any dog.

\section{RESULTS}

EVALUATION OF THE METHOD The accuracy of a dye dilution method in measuring small bowel motility depends upon the efficiency of collecting samples at the collecting site. The use of a Foley's catheter as described ensures this. The flow rates calculated from the dye dilution curves correlated closely with the flow rates determined independently by the ratio of PEG concentration in the infused solution to that in the pooled sample obtained from the jejunal cannula $(\mathrm{r}=0.98, \mathrm{P}<0.001)$.

EFFECT OF METOCLOPRAMIDE Table $I$ is a summary of the results. In each dog metoclopramide (drug A) caused a significant decrease in the mean transit time and volume of the perfused segment in comparison with B (saline) and saline. The mean flow rates were not significantly different. The dye dilution curve after the injection of metoclopramide reached an earlier and higher peak than when saline was injected (Fig. 2). However, the areas were almost exactly equal, confirming the similarity of flow rates at the collection site.

\section{DISCUSSION}

The method described to measure flow rate and volume interrelationships in the small intestine is identical in principle to the Stewart-Hamilton technique for measuring cardiac output (Dow, 1956). However, in the intestine, the dye does not recirculate, thus eliminating the need to extrapolate the dye dilution curve to the baseline. The technique is only applicable to 'steady-state' conditions, and, for this reason, a steady perfusion of the small intestine had to be created by constant infusion of an isotonic electrolyte solution.

The validity of a dye dilution method for measuring small bowel motility also depends on the constancy of flow past the collection site. If flow rate changes significantly during the collection of samples for a dye dilution curve, calculation of transit time, flow rate, and volume will not be valid. However, if flow rate does change during collection of samples for a curve this will be evident on inspection of the curve. A dye dilution curve characteristically exhibits an exponential fall off in dye concentration after the peak is reached provided there is uniform mixing and wash out (Dow, 1956). Invalid curves can be recognized by the absence of an exponential fall off in dye concentration.

The results of our study indicated that the method can be used to give a satisfactory measure of transit, flow rate, and volume in a segment of small intestine. We incorporated a second non-absorbable dye (PEG) in the test solution in order to check the validity of using bromsulphalein. Although the results using BSP gave a slightly higher value for flow rate than did PEG, the correlation between the results with two materials was very high. Since PEG was measured only in pooled samples, calculations of transit time could not be made using this material. However, because the correlation between the PEG and the BSP results was high, the BSP results could be used to assess the effect of drugs on the variables measured.

Metoclopramide resulted in a decrease in the mean transit time and volume of the perfused segment of intestine, while the flow rate remained unchanged. The decrease in volume of the perfused segment could be due to a decrease in bore or length, or both. These results are compatible with the study in vitro by Eisner (1968), in which metoclopramide resulted in contraction of both longitudinal and circular strips of human small intestine.

We are glad to have this opportunity to express our gratitude to Mr E. Gains, Miss S. Harris, Miss G. 
Wallbridge, Miss M. McDermott, and Mrs Delores Rigby for their help.

\section{REFERENCES}

Dillard, R. L., Eastman, H., and Fordtran, J. S. (1965). Volume-flow relationship during the transport of fluid through the human small intestine. Gastroenterology, 49, 58-66.

Dow, P. (1956). Estimations of cardiac output and central blood volume by dye dilution. Physiol. Rev., 36, 77-102.

Eisner, M. (1968). Gastrointestinal effects of metoclopramide in man. In vitro experiments with human smooth muscle preparations. Brit. med. J., 4, 679-680.

Hakuhara, T., Nakayama, S., Fakuda, H., Siina, N., and Neya, T. (1966). Effects of metoclopramide on the movements of the stomach and small intestine in dogs. Jap. J. smooth Muscle Res. 2, 15-21.
Hyden, S. (1955). A turbidometric method for the determination of higher polyethylene glycols in biological materials. $K$. LandtbrHogsk. Annlr, 22, 139-147.

Jacoby, H. I., and Brodie, D. A. (1967). Gastrointestinal actions of metoclopramide. Gastroenterology, 52, 1, 676-684.

James, W. B., and Hume, R. (1968). Action of metoclopramide on gastric emptying and small bowel transit time. Gut, 9, 203-205.

Justin-Besancon, L and Laville, C. (1964a). Action anti émétique du métoclopramide vis-a-vis de l'apomorphine et de l'hydergine. C.R. Soc. Biol. (Paris), 158, 723-727.

(1964b). Action du métoclopramide sur le systeme nerveux autome. Ibid., 158, 1016-1018.

- $\longrightarrow$, and Thominet, M. (1964). Le métoclopramide et ses homologues. Introduction à leur étude biologique. C.R. Acad. Sci (Paris), 258, 4384-4386.

Zierler, K. L. (1958). A simplified explanation of the theory of indicator-dilution for measurement of fluid flow and volume and other distributive phenomena. Bull. Johns Hopk. Hosp., 103 199-217. 